\title{
Divided PE and HDF Saved a Patient with Acute Pancreatitis Caused by Hyperlipidemia in the Final Stage of Gestation
}

\author{
Nobuhisa MATSUHASHI ${ }^{12)}$, Kazunori YAWATA ${ }^{1)}$ and Shinji OGURA ${ }^{1)}$ \\ ${ }^{1)}$ Department of Emergency \& Disaster Medicine, Gifu University \\ ${ }^{2)}$ Department of Surgery, Gifu Prefectural General Medical Center
}

\begin{abstract}
Acute pancreatitis is rare during pregnancy with hyperlipidemia found in only about 100 cases reported in Japan. We used divided plasma exchange (PE) and hemodia-filtration (HDF) for treatment.

A 28-year-old pregnant woman was examined at 29 weeks of gestation. Laboratory data on admission revealed an increased white blood cell count of $16,300 / \mathrm{mm}^{3}$, total cholesterol $1,072 \mathrm{mg} / \mathrm{dl}$, triglycerides $4,288 \mathrm{mg} / \mathrm{dl}$, serum amylase $850 \mathrm{IU} / \mathrm{L}$, elastase $1,002 \mathrm{ng} / \mathrm{dL}$, and phospholipase A2 2,850ng/dL. The acute pancreatitis diagnosis with $\mathrm{CT}$ evaluation was grade IV. In addition, the Acute physiology and chronic health evaluation (APACHE) II score was 14 points, Sequential organ failure assessment (SOFA) score 7 points, Ranson score 4 points. First, we performed $\mathrm{PE}$ and $\mathrm{HDF}$, but filtration was impossible due to hyperlipidemia. Subsequently, we succeeded with our new method, divided PE and HDF. The patient was discharged from the hospital on the 17th hospital day. Both the patient and her baby are in good health. This is the first report of a new therapy for acute pancreatitis during pregnancy with hyperlipidemia. The experience of this case and a review of the English literature suggested that divided $\mathrm{PE}$ and HDF are recommended to treat this rare hyperlipidemia.
\end{abstract}

Key words: acute pancreatitis, hyperlipidemia, gestation

Received: September 28, 2009/Accepted: November 17, 2009

Correspondence to: Nobuhisa Matsuhashi

Department of Emergency \& Disaster Medicine, Gifu University, 1-1 Yanagido, Gifu City, Gifu 5011194, Japan

\section{Introduction}

Pancreatitis complicating pregnancy is uncommon, so most obstetricians have limited experience of managing such patients. The incidence varies from $1 / 1,066$ to $1 / 2,888$ of deliveries. Although pregnancy, especially in nulliparous women, was once regarded as a specific predisposing condition for pancreatitis, this concept was later rejected. In nonpregnant patients, gallstones and alcohol abuse are almost equal contributors to $80 \%$ of pancreatitis cases, whereas $10 \%$ are from a long list of causes and $10 \%$ are idiopathic.

There have been about 100 reports of pancreatitis complicating pregnancy in Japan. In addition, in prior reports, maternal and perinatal mortality rates of $20 \%$ to $50 \%$ were described. In this report, we describe a case of acute pancreatitis complicated by hyperlipidemia that occurred during the final stage of pregnancy, and we recommend our new method, divided PE and HDF, if filtration is impossible due to hyperlipidemia.

\section{Case Presentation}

The patient was a 28-year-old woman, gravid 2nd. She developed hyperlipidemia, as had her mother. The patient complained of nausea and abdominal pain in the 29th week of gestation. The pain gradually increased in the epigastric region. Laboratory examination showed abnormal levels of serum amylase $850 \mathrm{IU} / \mathrm{L}$, total cholesterol 1,072mg/dL, HDLcholesterol $21 \mathrm{mg} / \mathrm{dL}$, triglycerides $4,288 \mathrm{mg} / \mathrm{dL}$, elastase 1,002ng/dL, phospholipase A2 2,850ng/dL, calcium $6.9 \mathrm{mg} / \mathrm{dL}$, WBC $16,300 / \mathrm{mm}^{3}$, CRP $2.03 \mathrm{mg}$ / dl, PT $60 \%$, AT-III $61 \%$ with normal AST, ALT, LDH, ALP, BUN, Cr, Hb, PLT and glucose (Table 1 ).

The diagnosis was acute pancreatitis complicated 
PE and HDF saved a patient with acute pancreatitis

Table 1 Laboratory data on admission

\begin{tabular}{|c|c|c|c|}
\hline TP & $5.2 \mathrm{~g} / \mathrm{dl}$ & T-bil & $0.3 \mathrm{mg} / \mathrm{dl}$ \\
\hline Alb & $3.4 \mathrm{~g} / \mathrm{dl}$ & FBS & $126 \mathrm{mg} / \mathrm{dl}$ \\
\hline CK & $103 \mathrm{IU} / \mathrm{L}$ & CRP & $2.03 \mathrm{mg} / \mathrm{dl}$ \\
\hline AST & $<5 \mathrm{IU} / \mathrm{L}$ & WBC & $16,300 / \mu l$ \\
\hline ALT & $13 \mathrm{IU} / \mathrm{L}$ & $\mathrm{RBC}$ & $398 \times 10^{4} / \mu 1$ \\
\hline $\mathrm{LDH}$ & 202 IU/L & $\mathrm{Hb}$ & $14.0 \mathrm{~g} / \mathrm{dl}$ \\
\hline ALP & $217 \mathrm{IU} / \mathrm{L}$ & $\mathrm{HCT}$ & $35.4 \%$ \\
\hline$\gamma$-GTP & $14 \mathrm{IU} / \mathrm{L}$ & PIt & $24.2 \times 10^{4} / \mu \mathrm{I}$ \\
\hline AMY & $850 \mathrm{IU} / \mathrm{L}$ & PT & full limits \\
\hline $\mathrm{Cr}$ & $0.54 \mathrm{mg} / \mathrm{dl}$ & APTT & full limits \\
\hline BUN & $6.3 \mathrm{mg} / \mathrm{dl}$ & FDP & $4.8 \mu \mathrm{g} / \mathrm{ml}$ \\
\hline TG & $4,288 \mathrm{mg} / \mathrm{dl}$ & $\mathrm{pH}$ & 7.35 \\
\hline T-chol & $1,072 \mathrm{mg} / \mathrm{dl}$ & $\mathrm{pO}_{2}$ & $93 \mathrm{mmHg}$ \\
\hline $\mathrm{Na}$ & $121 \mathrm{mEq} / \mathrm{L}$ & $\mathrm{pCO}_{2}$ & $23 \mathrm{mmHg}$ \\
\hline K & $3.5 \mathrm{mEq} / \mathrm{L}$ & $\mathrm{CHCO}_{3}^{-}$ & $12.4 \mathrm{mmol} / \mathrm{L}$ \\
\hline $\mathrm{Cl}$ & $90 \mathrm{mEq} / \mathrm{L}$ & cBase & $-11.2 \mathrm{mmol} / \mathrm{L}$ \\
\hline $\mathrm{Ca}$ & $6.9 \mathrm{mEq} / \mathrm{L}$ & & \\
\hline
\end{tabular}

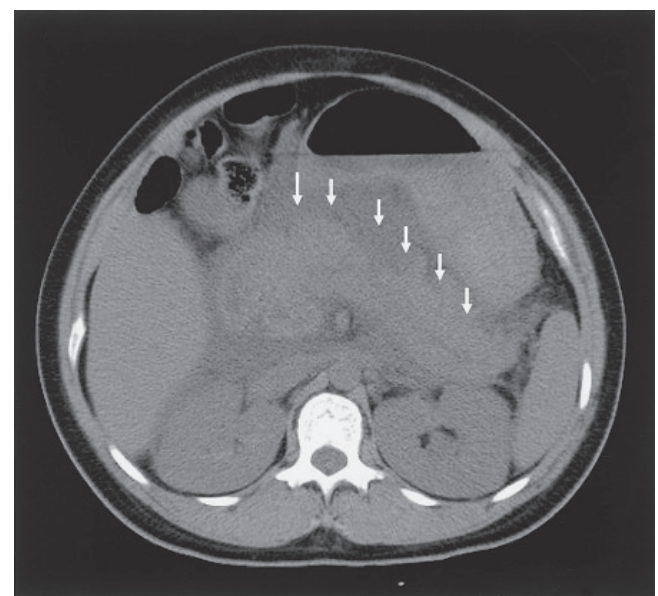

Fig. 1 Noncontrast CT showed peripancreatic inflammation from head to tail and fluid collection (arrow head).

by hyperlipidemia. On admission, her blood pressure was $120 / 80 \mathrm{mmHg}$ and her pulse was regular at 120 beats/min. Her consciousness was 2-2-6 on the Glasgow coma scale. In addition, the severity score of pancreatitis was 9 points and CT grade IV (Fig.1, 2). The APACH II score was 14 points, SOFA score 7 points, and Ranson score 4 points.

The patient was intubated and treated in the intensive care unit (ICU) of our hospital. We implanted an infusion catheter for continuous arterial infusion (CAI) of a protease inhibitor and imipenem cilastatin sodium for acute pancreatitis.

The infusion catheter was placed into the gas-

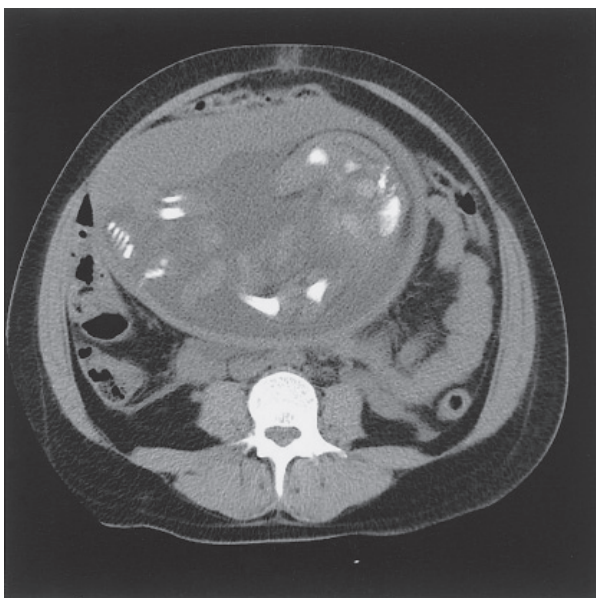

Fig. 2 Noncontrast CT showed the fetus in utero.

troduodenal artery via a femoral artery approach. In addition, we infused a vascular access system (VAS) and performed plasma exchange (PE) and hemodia-filtration (HDF) (Fig. 3 ), but initially we could not perform PE and HDF because her hyperlipidemia blocked the column filtration. We tried to repeat $\mathrm{PE}$ and $\mathrm{HDF}$ using dialysis liquid for preload purification (Fig. 4 ), but they could not be completed due to a blocked column; therefore, blood and plasma were separated, the blood cells were returned, and plasma was transfused (Fig. 5 ). As a result, the patient gradually improved and was discharged from the hospital on the 17th hospital day. Both the patient and her baby are in good health (Fig. 6 ).

\section{Discussion}

Acute pancreatitis can be caused by various factors such as alcohol, drugs, cholelitiasis, hyperparathyroidism, and hyperlipidemia. The onset of acute pancreatitis during pregnancy is rare, with an estimated incidence of $0.03 \%$ in Japan. Less than $10 \%$ of all cases of acute pancreatitis occurring during pregnancy are associated with cholelitiasis, while in Europe and North America, cholelitiasis is found in about $50 \%$ of $\operatorname{cases}^{1 \sim 3)}$. Several theories have been proposed to explain the mechanism of acute pancreatitis during pregnancy. Abdominal pressure due to pregnancy may constrict the duodenal mucosa, biliary tract, and the sphincter of the Oddi muscle, resulting in bile retention. On the other hand, hyper- 


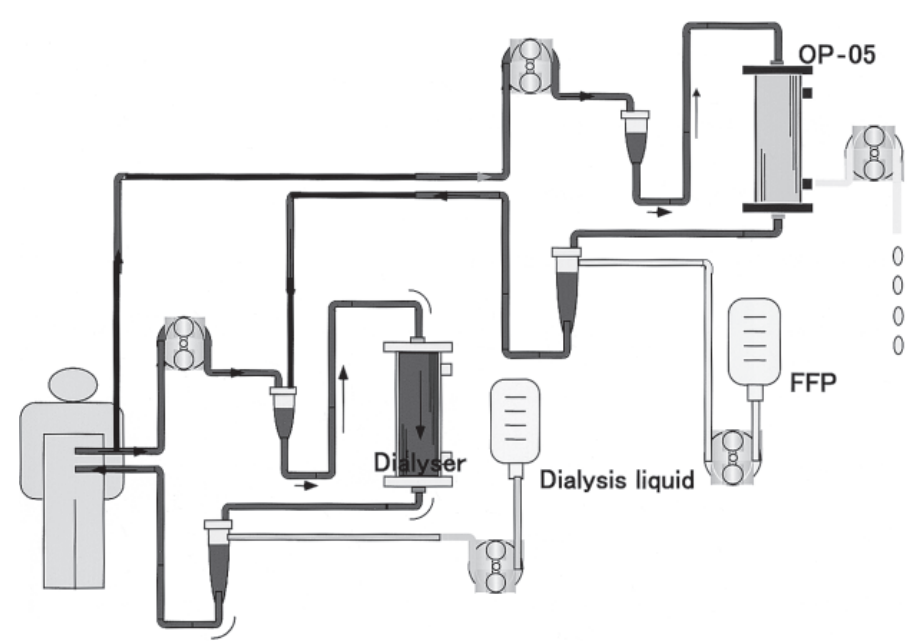

Fig. 3 The normal process of PE and HDF.

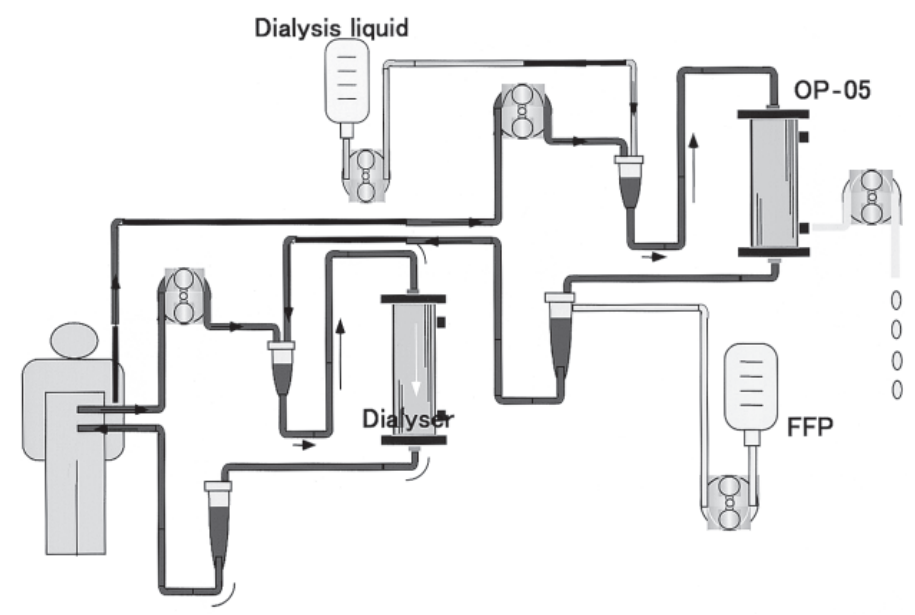

Fig. 4 Another process of PE and HDF using pre-load dialysis liquid.

lipidemia may be induced physiologically in pregnant women; plasma lipid levels begin to rise from the third month of pregnancy, reaching peak levels in the $33^{\text {rd }}$ week. Yoshioka and Eguchi ${ }^{4)}$ reported that hyperlipidemia occurs in $31.3 \%$ of pregnant women during the final stage of pregnancy, with serum cholesterol levels 0.5 to 1.5 times higher and serum triglyceride levels 1.5 to 2.5 times higher than the respective values in non-pregnant women.

In our case, in the 29th week of gestation, laboratory examination showed very high levels of serum amylase $850 \mathrm{IU} / \mathrm{L}$, total cholesterol 1,072mg/dl, and triglycerides $4,288 \mathrm{mg} / \mathrm{dl}$.

Wilkinson $^{5)}$ reported maternal mortality of $37 \%$ and fetal mortality of $37.9 \%$ in pregnancy complicated by pancreatitis. Acute pancreatitis in pregnancy is therefore more dangerous to the fetus than the mother. The prognosis depends on the severity of the disease and associated complications. As preterm labor may occur in as many as $60 \%$ of patients with pancreatitis in late pregnancy, gestation age is a primary determinant of prenatal outcome.

Therefore, the development of rapid enzyme assays leading to earlier diagnosis and improvement in both supportive and intensive care have no doubt also played a role in this decline in mortality.

Usually, blood purification for pancreatitis is performed by peritoneal perfusion and dialysis because 


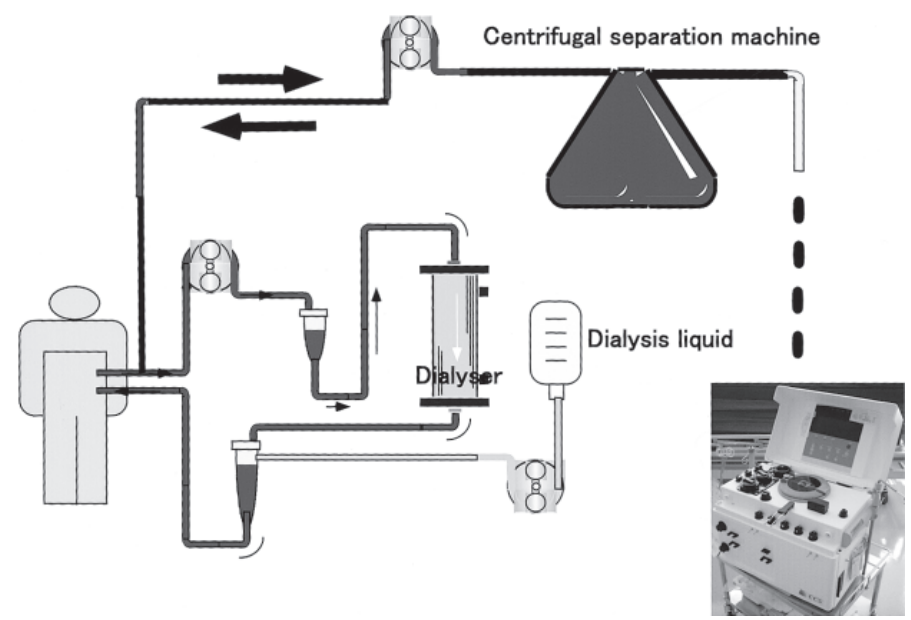

Fig. 5 Our new method of PE and HDF which uses centrifugal separation.

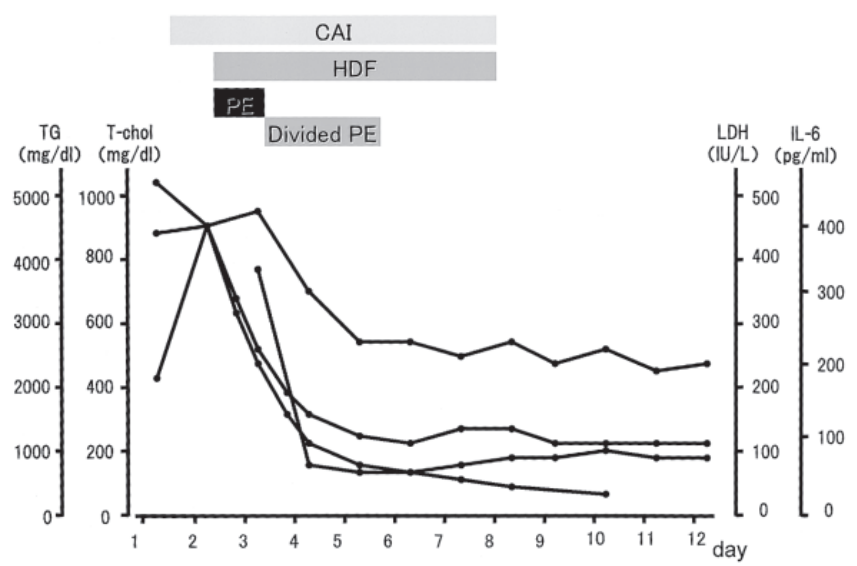

Fig. 6 Changes in the TG, T-cho, LDH and IL-6 levels during clinical course.

these techniques can directly remove pancreatic enzymes and humoral medicators such as cytokines from ascites, peritoneal macrophages primed by these factors, and necrotic debris present in the abdominal cavity.

In addition, HDF can efficiently remove from the blood most factors causing pancreatitis, such as enzymes released from the pancreas, for example, phospholipase A2 and trypsin, cytokines, and humoral mediators.

Therefore, one drawback of continuous hemodiafiltration (CHDF) is the risk of hemorrhagic complications due to anticoagulation. These complications indicate gastrointestinal bleeding and recurrent hemorrhage, but HDF has less risk of hemorrhage than CHDF.

Our new method, divided PE and HDF, was performed because the HDF filtration column was blocked due to hyperlipidemia. Blood and plasma were separated by centrifugal separation, the blood cells were returned and plasma was transfused. As a result, HDF could remove phospholipase A2, trypsin, cytokines and humoral mediators.

The patient was discharged from the hospital on the 17th hospital day. Both the patient and her baby are in good health.

In this report, we described a case of acute pancreatitis complicated by hyperlipidemia that oc- 
curred during the final stage of pregnancy, and we recommended the use of a new purification method.

\section{References}

1) Trapnell JE, Duncan EHL : Paterns of incidence in acute pancreatits : Clinical review. Br Med J 26 : 179-183, 1975

2) Berk JE, Smith BH, Akrawi MM : Pregnancy pancreatitis. Am J Gastroenterology 56 : 216-226, 1971
3) Ramin KD, Ramin SM, Richery SD, et al : Acute pancreatitis in pregnancy. Am J Obstet Gynecol 176 : 187-191, 1995

4) Yoshioka T, Eguchi K : Pregnancy and hyperlipidemia. Obstet Gynecol Practice 26 : 431-435, 1977

5) Wilkinson EJ : Acute pancreatitis in pregnancy : A review of 98 cases and areport of 8 new cases. Obstet Gynecol Surv 28 : 281-303, 1973 